rence in the lymph glands alone. In only one case did recurrence make its appearance at a period of more than three years after the operation."

George W. Crile, writing in 1905, 1906 and 1907 in different periodicals, advocates what he calls the "block" operation for cancer of the mouth and neck. There is time for but a brief reference to this classic work which was destined to revolutionize neck surgery. The author boldly takes the ground that all the lymphatic and other tissues containing possible cancer cells should be removed, and points out the parallelism in cancer of the breast where success first attended operative interference when the radical Halstead operation was introduced. In reviewing the literature, Crile found that in 4,000 cases, but 38 , or less than $1 \%$, had distant metastases, showing that cancer cells rarely get below what he calls the "collar" which the lymph nodes form about the neck. Taking advantage of this fact and that the structures of the neck though complicated are so easily accessible, he considers that the field is one particularly favorable to a total extirpation of the disease. His operative results justify this contention. He began the radical method in 1900. Since then he had operated on 122 cases, with a mortality of only $8 \%$. Of these, the cases in which the radical operation was done show a mortality only one quarter of that where the old method was deemed sufficient. With perfection in technic the death-rate lessened, for he had had no deaths in the last twenty cases. He finds that the nature of the operation must be largely determined by experience, and that each case must be considered individually. His own researches, and those of Porier, Kuttner and others, have thrown new light on the lymphatics of this region. If the disease is on one side, it almost invariably stays on that side, and goes to given and definite lymph nodes. If the tumor is in the median line, then it may go to one side or the other, and the operation is determined accordingly. Briefly, he tries to keep just in advance of the process. If, for instance, the cancer involves only the tonsil, he removes also the superior deep cervical nodes, while if he finds one of these glands enlarged, he removes all the glands on that side of the neck. Inasmuch as the deep lymphatic system is so closely associated with the venous, he generally removes the internal and external jugular on that side. The excised block, which he starts removing from below, then includes "fascia, fat, the salivary and part of the parotid gland, the sterno-mastoid and omohyoid muscles and part of the stylohyoid and digastric, the entire venous system, and all of the lymphatics and glands in this region." The deep nerves and arteries and muscles are not molested.

Crile found that $50 \%$ of the old cases died of local or pulmonary infection. To avoid this, in cases where the respiratory tract must be entered from without, he makes a preliminary tracheotomy, thus walling off the mediastinum by granulations and keeping the operative field separate. He cares for the danger from hemorrhage by clamping the carotid and by regulating the blood pressure with his pneumatic suit. He avoids many cases of shock and exhaustion by careful examination prior to the operation. He further guards against trouble by a careful development of his technic. Some of his maxims are: Avoid blunt dissection and tearing, handling of diseased tissues and vigorous and repeated sponging; retract only on healthy tissues; use sharp dissection where possible; keep outside of diseased tissue; treat possible cancer tissue as you would a virulent infection, keeping it from contact with the healthy tissue.

Our first thought is that too many important structures of the neck are removed. But Crile finds that the deep muscles of the neck are easily able to take care of the motion of the neck, and that the venous circulation is readily and quickly compensated. In one case, he removed the internal and external jugular veins on one side. Later he was obliged to do a "block" operation on the other side. He found the small anterior veins dilated to the size of the old jugular vein and easily able to take care of its return flow. So he did not hesitate to resect the internal and external jugular veins on the opposite side, nor did the patient suffer any embarrassment, though all the jugulars were then resected.

His conclusions are "that since the head and neck present an exposed field, cancer here, unlike that of the stomach, the intestines or even the breast, may be recognized at its very beginning; that every case is at some time curable by complete excision; that the field of regional metastases is exceptionally accessible; that cancer rarely penetrates the extraordinary lymphatic collar of the neck; that the growth tends to remain localized here; that by applying the same comprehensive dissection as in radical cure of cancer of the breast, and by freely utilizing the modern researches of surgery, the final outcome in cases of cancer of the neck and head should yield better results than that of almost any other portion of the body."

In the particular case brought to your attention this evening, the question may arise as to whether this patient, who has since passed her eighty-sixth birthday, could have borne the radical neck operation. She has been relieved of much discomfort and her end will not be that which threatens such cases when not operated on.

In closing the writer would express his appreciation to Dr. John C. Berry and Dr. R. P. Watkins for being permitted to work with them on this case.

\section{TWO CASES OF HYPERTROPHIC STENOSIS IN INFANTS.*}

BY C. A. PORTER, M.D.,

Surgeon to the Massachusetts General Hospital.

CASE I. C. E. H. Entered Massachusetts General Hospital in my service on Aug. 10, 1909. Aged five weeks. Mother in excellent health; one other child. Normal delivery at the Lying-In Hospital, Boston;

* Read at a meeting of the New England Pediatric Society, Oct. 21 
weight, $7 \mathrm{lb}$. Took breast with occasional slight regurgitation; stools daily and yellow in color. Mother and boy discharged well in two weeks.

Two weeks before entrance, baby began to vomit after some feedings; no bile in vomitus; constipation. Gradually vomiting increased in frequency and the child was admitted to the Infants' Hospital. Breast feedings continued, as did the vomiting. Milk sugar given without benefit. Examination a week before entrance showed two small masses just below the ensiform cartilage. Bowel distended with salt solution, which filled up the epigastric region. For three days the baby was placed on rectal feedings, peptonized milk and brandy. Vomiting continued; stools pasty and yellow-brown. Sent to the Massachusetts General Hospital by Dr. Whitehouse with diagnosis of probable intussusception.

Examination. - Emaciated baby which looks starved but not sick. Pulse good quality; tongue moist with white coat; slight aphthæ. Heart and lungs negative. Abdomen full; very slightly distended; tympanitic; no visible mass nor evidences of peristalsis. Under the right rectus muscle, just below the costal angle, a round mass can be felt about, the size of a marrow-fat pea, which is freely movable, especially toward the median line and downward. Stomach shows no visible distention; anus negative; no enlarged glands.

Seen by Drs. F. T. Lord, J. L. Morse, A. K. Stone and myself. Given rectal salt solution, 2 oz., every four hours with four drops of brandy; boiled water by mouth, $\frac{1}{2}$ oz. every four hours. Baby vomited the water occasionally, but retained the brandy and rectal salt solution.

Dr. Frederick T. Lord's consultation blank reads as follows: "The history of propulsive vomiting, continuous for two weeks, with the presence of visible peristalsis and an elongated tumor in the right hypochondrium, are, it seems to me, sufficient to make the diagnosis of pyloric stenosis. As there has been fecal matter by rectum throughout illness, the stenosis is probably incomplete."

On Aug. 11, the baby was seen by Dr. J. L. Morse, who could feel a small lump deep in the right upper quadrant. No apparent distention of the stomach; no visible peristalsis. Stomach tube passed and $3 \mathrm{oz}$. of boiled water put in; most of this retained by the baby. Dr. Morse did not consider the diagnosis of pyloric obstruction well proven and advised further observation. He advised human milk diluted one third, or whey mixture $\frac{1}{2}$ oz. every two hours. The baby retained the first feeding of whey, but vomited the first mother's milk, retaining, however, the second feeding; condition unchanged.

On Aug. 13, definite peristaltic waves developed in the stomach, and Dr. Morse made the diagnosis of pyloric obstruction on the same evidence which Dr. Lord had on Aug. 10.

Operation. - Child wrapped in cotton batting, except for abdomen. Two-inch median incision above umbilicus; stomach found to be dilated, with a definite hypertrophic ring at the pylorus, about the size of the end phalanx of the little finger. No small stomach clamps were at hand, so a posterior gastro-enterostomy was done without clamps, using the finest Pagenstecher for the peritoneum and fine silk for the mucosa. The wound was closed with through-and-through interrupted sutures. Whole operation, thirty-five minutes. Patient sent to the ward in fair condition, but soon cyanosis developed, which improved rapidly with oxygen inhalations. Boy given boiled water, $2 \mathrm{dr}$., every two hours at once; in addition, salt by rectum and subpectorally.

On Aug. 14, general condition improved.
On Aug. 15, modified milk 2:5:25 and 10\% lime water, which it took hungrily; though vomiting some feedings, the majority of them were retained.

On Aug. 16, child considerably better. Two brownish stools in morning; in the afternoon there developed a green, slimy diarrhea, flecked with blood. Milk of bismuth $\frac{1}{2} \mathrm{dr}$. given every hour until the afternoon, when only boiled water was allowed. By evening, the diarrhea had not abated, and the child had had fourteen movements.

Aug. 17, child took mother's milk 1-3 greedily with brandy m.v every two hours; salt solution by rectum; eleven slimy green dejections.

On Aug. 17, six days after operation, the dejections during the day had fallen to five. Mother's milk given undiluted; baby brighter; subpectoral salt continued.

On the 20th, six movements during night and five during day. Baby took $6 \mathrm{dr}$. of milk every one and one-half hours, and did not vomit. Temperature normal this morning; pulse, 130; respirations, 28.

During the night baby had six dejections and in the morning (the 21st) did not seem as bright; pulse was poor. During the day, the child failed rapidly, the diarrhea continued, and the child died at two in the afternoon, eight days after the operation. Abdominal wound nearly healed.

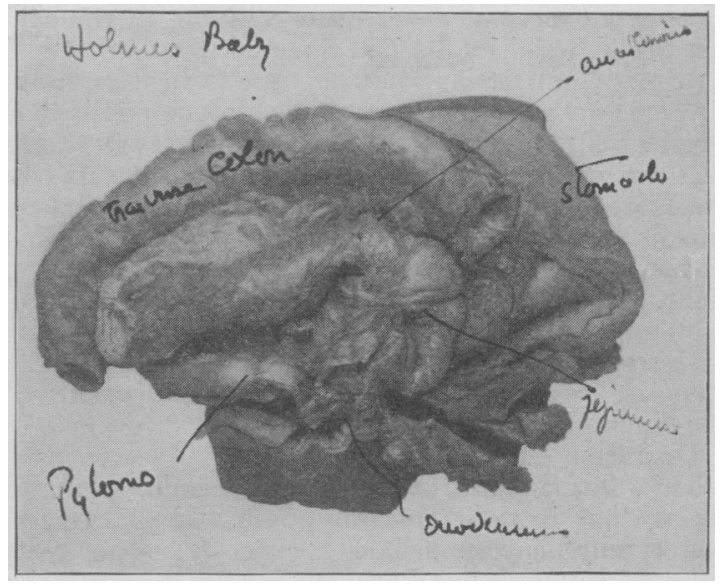

Autopsy specimen nine days after operation.

AUTOPSY 2424

Autopsy eighteen hours post mortem. Male infant six weeks old; $59 \mathrm{~cm}$. long; well developed; emaciated; head not examined.

Trunk. - In the median line of abdominal wall is a linear wound $5 \mathrm{~cm}$. long, closed with sutures, which extend from umbilicus directly upward. Subcutaneous fat small in amount; muscles thin and pale. The peritoneal cavity contains a very small amount of thin bloody fluid. The anterior margin of the right lobe of the liver is at the costal border in the right mammary line.

Pleural cavities and lungs normal. Thymus gland present but not remarkable.

Heart and pericardium not remarkable.

Stomach slightly dilated and an anastomosis established between stomach and upper end of jejunum at posterior root; anastomotic opening free. The pyloric end of the stomach over a length of $4 \mathrm{~cm}$. is tubular in shape; walls thickened; on section they are at least $5 \mathrm{~mm}$. in thickness; thickening apparently mostly in muscular coat; lumen of pylorus greatly reduced, about 2.5 in diameter. Stomach otherwise not remark- 
able. Small and large intestine on section show nothing to record. No peritonitis.

Bacteriological examination of heart's blood show one small, round, raised, yellowish, opaque colony on the surface, showing large gram-staining cocci. Water of condensation, no growth.

Case II. L. H. S., Hyannis, Mass.; boy of ten weeks. Referred by Dr. Wm. J. Brown, of 1080 Boylston Street, to the Massachusetts General Hospital on June 10, 1911, in my service. Diagnosis made by Dr. Brown of pyloric stenosis.

History. - First child; full term; normal labor; weight $9 \frac{1}{2} \mathrm{lb}$. at birth. Boy seemed to be all right up to age of two weeks, having nursed at the breast well. Vomiting then began after each nursing. This continued until five weeks ago, when he seemed to vomit evervthing he took immediately after nursing. For past five weeks has vomited one to five times a day, but seemed to retain his food longer. For che two days previous to admission, vomited everything immediately after feeding; no blood nor bile in vomitus. Up to two weeks ago at breast for fifteen minutes at a time; since then only five minutes. Three weeks ago put on barley water alone for one week, but vomited just the same. Condensed milk diluted with water tried without benefit. During the first two weeks bowel movements daily of normal color, but since then the stools have been greenish, occasionally hard and curdy for several days at a time, but never watery. Now, at the age of ten weeks, weighs $9 \mathrm{lb}$., half a pound less than at birth. At times vomiting is projectile; at others, regurgitant.

Examination. - Fairly well-developed, poorly nourished child; fontanelles open and depressed; tongue and throat negative; mucous membranes of fair color; no enlarged glands; heart and lungs normal. Abdomen sunken and held somewhat rigidly. Stomach markedly distended, with active peristaltic waves passing to right. In the right hypochondrium is a mass the size of the end of little finger which is not tender and moves with respiration; no hernia. Diagnosis made of pyloric stenosis. Seen by Dr. R. M. Smith, who concurred in the diagnosis.

Operation June 13, 1911; ether.

Dr. John Homans looked after the child's condition, seeing that it was kept warm, etc. Median incision above umbilicus three inches long. Posterior gastroenterostomy with fine Pagenstecher for the peritoneum and chromic 00 catgut for the mucosa. One clamp was used on the stomach, but the collapsed jejunum did not require any clamping, and I found the operation easier without this other clamp. No examination was made of the tumor, as my endeavor was to do the operation as quickly as possible. Again the time was thirtyfive minutes.

Patient made a good recovery from ether. Temperature rose the night of operation to 102 and pulse 130 , but on the following morning temperature was 104 and the pulse 150 ; by evening, however, the temperature went down to normal and the pulse to 110 . No more fever up to time of discharge ten days after operation. The wound healed by first intention. The child was immediately placed in Dr. Fritz Talbot's care, who managed the feedings throughout, and has seen the case since then. I am told by him that the boy is now perfectly well and weighs $21 \mathrm{lb}$, a complete physical examination shows a perfectly normal bottle baby.

It seems reasonable to conclude that Baby $H$., the first case, died of an infectious midsummer diarrhea. Careful examination of the anastomosis and its surroundings showed no evidence of infection, and the opening was adequate. It is of interest to note that both of these children had apparently normal digestion for a period after birth, suggesting that with the development of the stomach the obstruction increased. While in the first case, it might have been possible to do a plastic operation on the pylorus, owing to the fact that the lumen was $2.5 \mathrm{~mm}$. in diameter, it seems to me that posterior gastro-enterostomy is the safest method of procedure, for there must undoubtedly be cases in which a plastic is impossible, and I do not know of any way of determining this from inspection without incision.

I was much impressed with the great importance of keeping these small children warm and dry during the operation, and with the necessity of immediate feeding after it. Although the parts to be sutured were necessarily small, neither operation was as difficult as I had feared.

\section{ACUTE MASTOIDITIS COMPLICATED BY TIC DOULOUREUX.*}

BY GEORGE H. POWERS, JR., M.D., BOSTON.

THE patient, a strong, previously healthy young man of twenty-eight, was first seen Jan. 7, 1911, suffering from "grippe" for the past week, with daily chills, temperature, headache, general malaise and a more or less constant severe pain in the right ear for three days. The drum membrane was red and bulging, with very acute mastoid symptoms. Temperature was $101 \mathrm{~F}$. The drum membrane was freely incised and the patient put to bed with orders for constant ice-coil, three-hour syringing, free catharsis and restricted diet. During the night there was a great deal of pain in and around the ear, above, behind and over the mastoid. In the morning the pain had markedly subsided, but on gently wiping the canal the patient suffered a severe paroxysm of pain in the above outlined region which lasted about twenty minutes. There was a copious sero-hemorrhagic discharge from the tympanum, and little change in the mastoid symptoms. About 5 P.M. the pain began and increased in severity until 9 P.M., when morphine was administered. No effect was perceptible until two hours later, in fact, subsequent doses of this drug for the relief of pain seemed to have but little effect and very much delayed at that.

During the following nine days the middle ear and mastoid infection progressed favorably, but the pain appeared daily about 5 P.M., and always upon wiping out the canal, however gently, although signs of a furuncle or local skin irritation were lacking.

From Jan. 17 to Jan. 20 the temperature was normal, middle ear discharge markedly less and mastoid tenderness entirely absent, but the afternoon and evening pain persisted, although less in severity. On Jan. 21 the pain was much increased, temperature rose to $102 \mathrm{~F}$. and mastoid symptoms returned with edema of the canal wall and over the tip. Dr. E. A. Crockett saw the case in consultation and was impressed by the severe paroxysm of pain which followed a gentle wiping of the canal, lasting as usual fifteen to twenty minutes. On the following day the mastoid was operated upon and found of the small-celled type, with the exception of one large cell in the tip, all cells being filled with pus (culture: streptococcus). No feature of especial interest presented itself during operation.

Convalescence progressed rapidly and uninterruptedly with reference to the wound and middle ear, the latter being dry on the sixth day and the mastoid wound completely healed in a little over four weeks.

* Read at a meeting of the New England Otological and Laryngological Society, Nov. 14, 1911. 